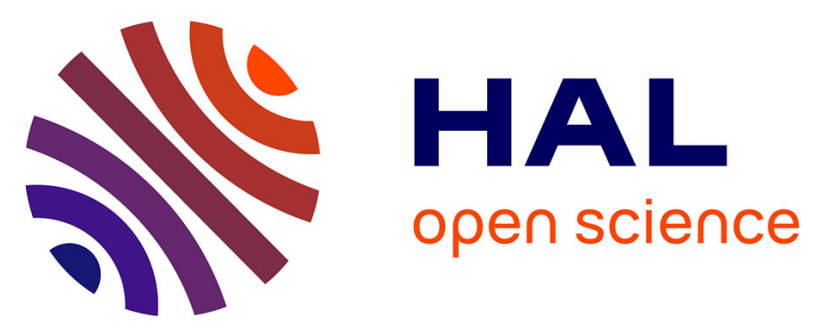

\title{
Modeling and characterization of an electromagnetic system for the estimation of Frequency Response Function of spindle
}

David Tlalolini, Mathieu Ritou, Clément Rabréau, Sébastien Le Loch, Benoît Furet

\section{To cite this version:}

David Tlalolini, Mathieu Ritou, Clément Rabréau, Sébastien Le Loch, Benoît Furet. Modeling and characterization of an electromagnetic system for the estimation of Frequency Response Function of spindle. Mechanical Systems and Signal Processing, 2018, 104, pp.294-304. 10.1016/j.ymssp.2017.11.003 . hal-01819017

\section{HAL Id: hal-01819017 https://hal.science/hal-01819017}

Submitted on 3 Jul 2018

HAL is a multi-disciplinary open access archive for the deposit and dissemination of scientific research documents, whether they are published or not. The documents may come from teaching and research institutions in France or abroad, or from public or private research centers.
L'archive ouverte pluridisciplinaire HAL, est destinée au dépôt et à la diffusion de documents scientifiques de niveau recherche, publiés ou non, émanant des établissements d'enseignement et de recherche français ou étrangers, des laboratoires publics ou privés. 
Modeling and characterization of an electromagnetic system for the estimation of Frequency Response Function of spindle.

\author{
David TLALOLINI ${ }^{\mathrm{a}}$, Mathieu RITOU ${ }^{\mathrm{a}}$, Clément RABREAU, ${ }^{\mathrm{a}, *}$, Sébastien LE LOCH ${ }^{\mathrm{a}}$, \\ Benoit FURET ${ }^{\mathrm{a}}$ \\ ${ }^{a}$ University of Nantes, LS2N (Laboratoire des Sciences du Numérique de Nantes, UMR CNRS 6004), \\ Nantes, France
}

\begin{abstract}
The paper presents an electromagnetic system that has been developed to measure the quasi-static and dynamic behavior of machine-tool spindle, at different spindle speeds. This system consists in four Pulse Width Modulation amplifiers and four electromagnets to produce magnetic forces of $\pm 190 \mathrm{~N}$ for the static mode and $\pm 80 \mathrm{~N}$ for the dynamic mode up to $5 \mathrm{kHz}$. In order to measure the Frequency Response Function (FRF) of spindle, the applied force is required, which is a key issue. A dynamic force model is proposed in order to obtain the load from the measured current in the amplifiers. The model depends on the exciting frequency and on the magnetic characteristics of the system. The predicted force at high speed is validated with a specific experiment and the performance limits of the experimental device are investigated. The FRF obtained with the electromagnetic system is compared to a classical tap test measurement.
\end{abstract}

Keywords: Electromagnetic force, Frequency Response Function, Machine tool, spindle dynamics

\title{
1. Introduction
}

The dynamic behavior of spindles has a great influence on the quality of machined parts, notably in the aircraft industry where instabilities in milling can lead to nonquality and reduced lifetime of spindle bearings [1] and cutting tool [2]. To improve the productivity of machine tools and to avoid the occurrence of chatter during milling, stability lobes diagrams are commonly used. The Frequency Response Function (FRF) at the tool tip is required and classically obtained by hammer impact test. It provides good results in classical milling. However, as shown in several studies $[3,4,5]$ the dynamic behavior of High Speed Machining spindles varies at high speed due to dynamic effects. Schmitz et al. [6] were the first to make measurements on a rotating spindle. Tap tests were performed at the tool tip, during spindle rotation. The displacement was measured with eddy-current sensors. In order to improve the measurement accuracy and

\footnotetext{
* Corresponding author

Email address: clement.rabreau@univ-nantes.fr (Clément RABREAU)
} 
repeatability, Albrecht et al. [4] used a specific tool with a ball bearing to make hammer impact test measurement on a fixed surface. Active Magnetic Bearings (AMB) were then used to apply non contact excitation to the spindle $[7,8]$ and to measure the cutting forces [9]. Rantatalo et al. [10] have presented a contact-less dynamic spindle excitation device that can apply a dynamic force of $\pm 15 N$ from 400 to $2000 \mathrm{~Hz}$. Matsubara et al. [11] succeeded to apply a dynamic force of $110 \mathrm{~N}$ from 0 to $1000 \mathrm{~Hz}$ with their electromagnetic system, however it presents non-linearities. Strong requirement are required in order to analyze the dynamic behavior of high speed spindles.

This paper presents an excitation device capable of applying a dynamic load of $\pm 80 \mathrm{~N}$ at up to $5000 \mathrm{~Hz}$. A specific current amplifier has been designed for this purpose. The control is linearized with the use of pre-magnetisation and an experimental protocol to measure the flux density at a large range of frequency is proposed. Thus, the applied force can be precisely estimated over the entire frequency range. First of all, the system design is described, then electromagnetic force models are developed for the static and dynamic excitation modes. The specific experiments carried out to validate the model and to update the permeability of the magnetic core are described. The dynamic characteristics of the excitation device are then studied. Finally, FRF measurement are made with the excitation system on a HSM spindle at several speeds and are compared with a classic tap test experiment.

\section{Description of the excitation system}

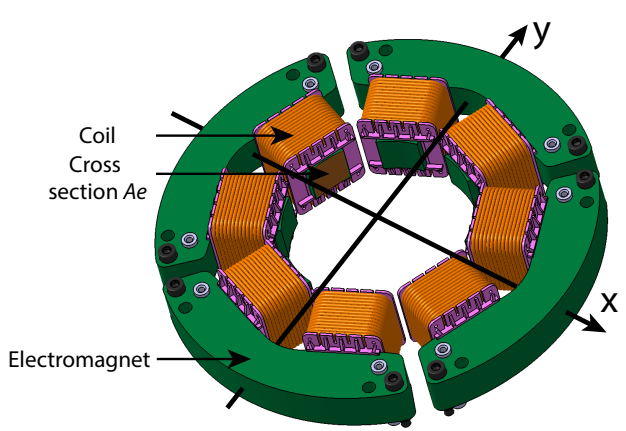

(a)

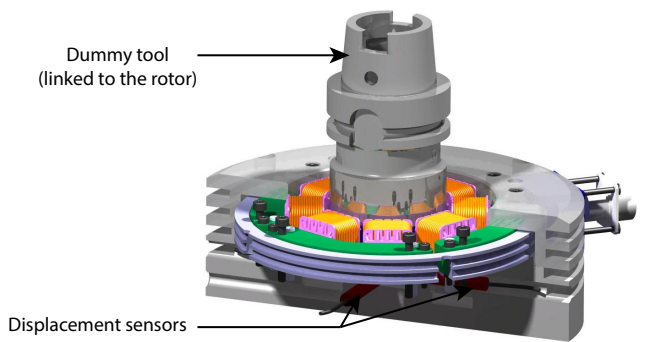

(b)

Figure 1: (a) The arrangement of the two pairs of electromagnets. (b) Sectional view of the excitation system and dummy tool.

The excitation system shown in figure 1, consists in a radial magnetic bearing with two perpendicular axes $(x$ and $y$ ) and a dummy tool with an HSK 63A interface for the mounting into the spindle rotor. Each axis is composed of two identical electromagnets with magnetic field of opposing directions to produce a radial force. A pre-magnetisation is applied in order to improve the performance and linearize the control. The system is designed to provide a magnetic force with amplitudes until $\pm 190 \mathrm{~N}$ for the static mode and $\pm 80 \mathrm{~N}$ for the dynamic mode. Each electromagnet is made of two coils of wire wrapped around a core of ferromagnetic steel sheets. The coils are wired in parallel one 
to each other in order to improve dynamic efficiency. The magnetic core is made from laminated sheet of grain oriented electrical steel (3\% Si-Fe) The core structure comprises 170 sheets with a thickness of $0.08 \mathrm{~mm}$ which are assembled shifting each oriented sheet from the previous one by an angle of $90^{\circ}$. A large cross section $A_{e}$ of the magnetic core was chosen. This configuration, studied in [12], gives the best performances concerning the magnetizing current, the core loss amount, the relative permeability and the reactive power. A stack of ferromagnetic steel sheets is also integrated in the dummy tool to strengthen the magnetic attraction.

\section{Theoretical model of the electro magnetic force}

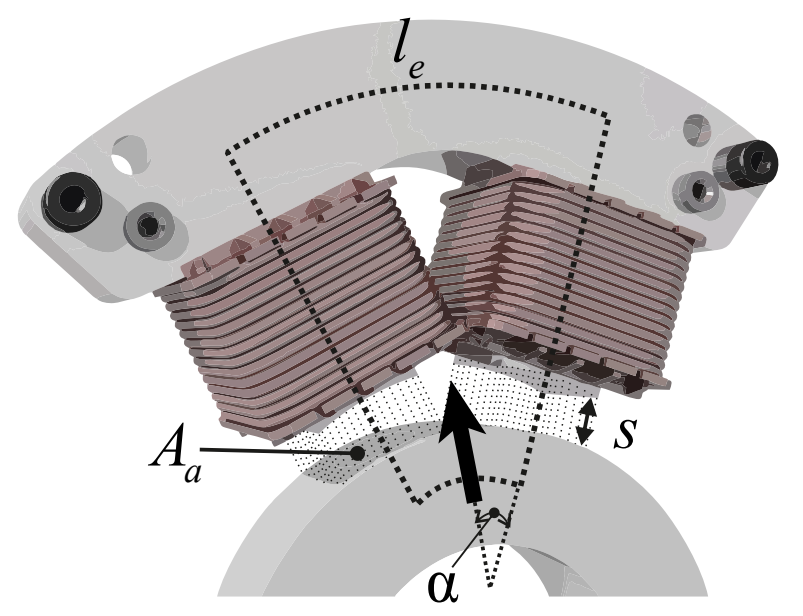

Figure 2: A two-coils electromagnet with magnetic cores made of stack of ferromagnetic steel sheets.

In order to determine the analytic force model, a single two-pole electromagnet is considered, as presented in figure 2. The figure illustrates the geometrical parameters of the electromagnet, i.e. effective magnetic path length $l_{e}$, air gap $s$ and effective crosssection area $A_{a}$ in the air gap. Since the air gap lengths are sufficiently small compared with their cross-sectional dimensions, the fringing flux effect can be neglected. In this consideration, the magnetic flux $\phi$ is homogeneous in the core $(c)$ and the air gaps $(a)$, i.e. $\phi=B_{c} A_{e}=B_{a} A_{a}$. Then, the flux density $B$ is the same along the path $l_{e}$ in the magnetic core $\left(B_{c}\right)$ and in the air gap $\left(B_{a}\right)$, i.e. $B_{a}=B_{c}=B$. Thus, the cross-sectional areas are the same $A_{a}=A_{e}$. Under these assumptions, the magnetic system can be assumed lossless and the magnetic force can be derived from the energy $W_{a}$ stored in the two air gaps, by

$$
W_{a}=\frac{1}{2} B H_{a} A_{e} 2 s
$$

where $H_{a}$ is the magnetic field intensity in one air gap, $A_{e}$ is the cross-sectional area and $s$ is the air gap length. The flux density $B$ is obtained through the permeability of 
free space $\mu_{0}: B=\mu_{0} H_{a}$. Let's consider a radial displacement $x$ of the shaft, along the radial force $f$. It involves a variation of the air gap of $x \cdot \cos (\alpha)$ (where $\alpha$ is the semi-angle between the two elementary forces, acting at each air gap). The force can be obtained from the principle of the virtual work $d W=f d x$. Assuming that $B$ remains constant, the magnetic force $f$ of the two-pole electromagnet derives from [13]:

$$
f=\frac{d W}{d x}=\frac{B^{2}}{\mu_{0}} A_{e} \cos (\alpha)
$$

\subsection{Static force model}

Considering a single two-pole electromagnet (as shown in figure 2), the static force model is derived from the magnetomotive force equations ( $\mathrm{mmf}$ ) generated by a current $i$ that is distributed in the two coils [14]:

$$
2 N i=\frac{l_{e} B}{\mu_{0} \mu_{r_{s}}}+\frac{2 s B}{\mu_{0}}
$$

where $N$ is the number of wire turns of the electromagnet coil and $\mu_{r_{s}}$ is the relative permeability of the magnetic core for the static mode. The first term of right part of the equation (3) describes the mmf due to the magnetic core and the second term is the mmf due to the two air gaps. The flux density $B$ is obtained by solving equation (3) in relation to the actual current $i$ and the air gap $s$ :

$$
B=\frac{2 \mu_{0} N}{\frac{l_{e}}{\mu_{r_{s}}}+2 s} i
$$

As mentioned before, each axis is composed of two identical electromagnets in a differential configuration that generate the magnetic force. This configuration is depicted in figure 3. According to equation (4), the static magnetic force of the axis $F_{s}$ can then be obtained as the difference of the forces of both electromagnets as shown in the following equation:

$$
F_{s}=f_{+}-f_{-}=4 \mu_{0} N^{2} A_{e} \cos (\alpha)\left(\frac{i_{+}^{2}}{\left(\frac{l_{e}}{\mu_{r_{s}}}+2 s_{+}\right)^{2}}-\frac{i_{-}^{2}}{\left(\frac{l_{e}}{\mu_{r_{s}}}+2 s_{-}\right)^{2}}\right)
$$

where $i_{+}$and $i_{-}$are the currents of each electromagnet. $s_{+}=s_{0}+x$ and $s_{-}=s_{0}-x$ are the respective instant air gaps for a radial displacement $x$ of the dummy tool and a nominal air gap $s_{0}$.

The magnetic force produced by each electromagnet shows the quadratic dependence on the current and the inversely quadratic dependence on the air gap. In order to linearize the control, a constant premagnetization current $i_{0}$ is applied. In addition, the control current $i_{c}$, leads to $i_{+}=i_{0}+i_{c}$ and $i_{-}=i_{0}-i_{c}$. Assuming that the system operates within its linear domain and that the displacement $x$ of the rotor is much less than the nominal air gap $s_{0}$, the static force can be simplified as:

$$
F_{s}=\frac{16 A_{e} \mu_{0} N^{2} \cos (\alpha)}{\left(\frac{l_{e}}{\mu_{r_{s}}}+2 s_{0}\right)^{2}} i_{0} \cdot i_{c}
$$


The value of $\mu_{r_{s}}$ is further obtained from experiments. The assumptions of linear domain and constant $B$ during a small displacement $x$ (for equation (2)) are verified in section 4.2 .

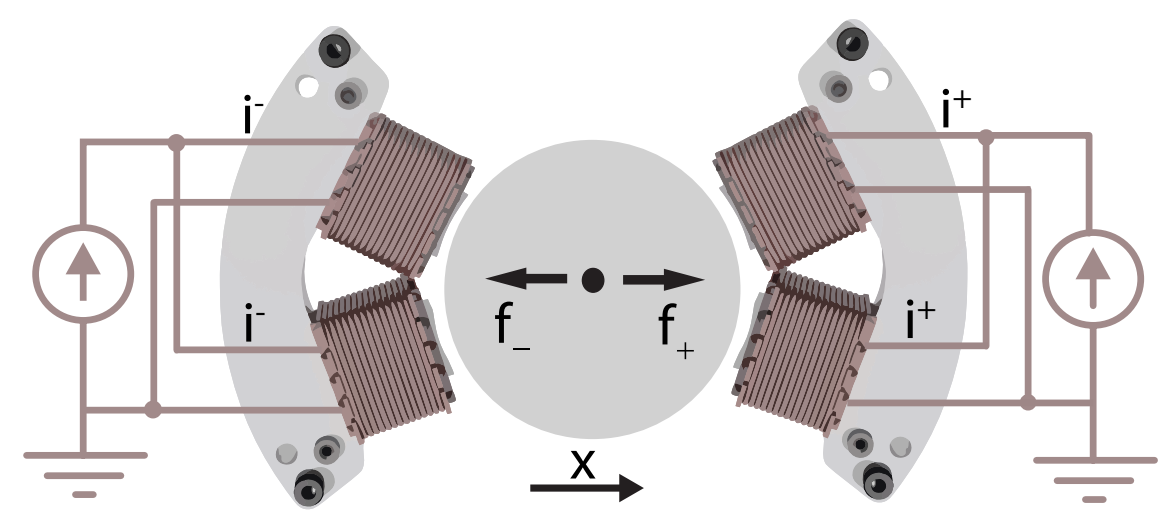

Figure 3: Specific arrangement of two electromagnets in order to achieve a linear control.

\subsection{Dynamic force model}

For harmonic excitation, the control current is sinusoidal, i.e., $i_{c}=\hat{I} \sin (\omega t)$. The variation of flux density closely approximate a sinusoidal function throughout the length of the core. Assuming that displacement $x$ is negligible (compared to $s_{0}$ ) and considering a premagnetization $i_{0}$, the magnetic flux in equation (4) can be written as a function of the sum $i_{0}+i_{c}$ and the difference $i_{0}-i_{c}$. Besides, the premagnetization flux $B_{0}$ and the dynamic flux $\hat{B}_{d}$ can be distinguished.

$$
\begin{gathered}
B=\frac{2 \mu_{0} N}{\frac{l_{e}}{\mu_{r}}+2 s_{0}}\left(i_{0} \pm i_{c}\right)=\frac{2 \mu_{0} N}{\frac{l_{e}}{\mu_{r_{s}}}+2 s_{0}} i_{0} \pm \frac{2 \mu_{0} N}{\frac{l_{e}}{\mu_{r_{d}}}+2 s_{0}} \hat{I} \sin \left(2 \pi f_{e} t\right) \\
B=B_{0}+\hat{B}_{d} \sin \left(2 \pi f_{e} t\right) \\
\text { with } \quad B_{0}=\frac{2 \mu_{0} N}{\frac{l_{e}}{\mu_{r_{s}}}+2 s_{0}} i_{0} \\
\text { and } \hat{B}_{d}=\frac{2 \mu_{0} N}{\frac{l_{e}}{\mu_{r_{d}}}+2 s_{0}} \hat{I}
\end{gathered}
$$

For one axis, the magnetic force $F_{d}$ for dynamic excitation is then:

$$
F_{d}=\frac{4 A_{e} \cos (\alpha)}{\mu_{0}} B_{0} \hat{B}_{d} \sin \left(2 \pi f_{e} t\right)
$$

$\mu_{r_{d}}$ is the dynamic relative permeability of the ferromagnetic core and depends on the pre-magnetisation current and on the frequency. The value is also different from the static $\mu_{r_{s}}$. 
The equation (7) enables the study of the behavior of the magnetic force under a sinusoidal excitation, but requires the flux density to be known. The determination of the flux density by means of an experimental test will be developed in the section 4.3. It will result in the identification of $\mu_{r_{d}}$.

\section{Experimental characterization of the electromagnetic system}

In this section, the experimental procedure to characterize the magnetic flux density, both for the static and dynamic modes, is described. For the static model, the flux density is determined from equations (4) and (6) and from the measure of the magnetic force. The flux density for the dynamic model will be estimated with a specific experimental set-up and measurement of induced voltage.

\subsection{Experimental set-up}

The experimental set-up, illustrated in figure 4 and 5, includes the excitation device mounted on a dynamometer table as well as the dummy tool clamped into the spindle rotor by an HSK 63A interface. The maximum spindle speed is $24000 \mathrm{rpm}$. The rotor is excited by the electromagnets with either Direct Current (DC) for quasi-static measurement or harmonic Alternating Currents (AC) for dynamic measurement. The excitation can be performed in both $x$ and $y$ radial directions. Due to the identical design of the $x$ and $y$ axes, the study is carried out in the $x$ axis only.

The excitation current, for each axis, is provided by two Pulse Width Modulation (PWM) amplifiers, specifically designed for this application and can provide a maximum continuous current of $15 \mathrm{~A}$ and $10 \mathrm{~A}$ rms per electromagnet in a frequency range from 50 to $5000 \mathrm{~Hz}$. In order to achieve this requirement, the amplifiers are designed with an internal current control loop. A non-contact displacement sensor is used to measure the instantaneous position of the rotor at the tip of the dummy tool. Other displacement sensors can be used to measure the displacement from another position of the dummy tool. For a DC excitation, the magnetic force is measured by a dynamometer table KISTLER 9255B. The National Instruments CompactDAQ system is used to interface the input and output analogic signals of the system at a sampling frequency $f_{s p}=50 \mathrm{kHz}$. A user interface was developed with LabVIEW software to manage the experiment. The measured datas are then converted and post-processed with Matlab.

\subsection{Static characterization and model identification}

A specific experiment was designed to characterize the static force behavior and to identify the relative permeability $\mu_{r_{s}}$. The experiment is carried out for one electromagnet without pre-magnetisation. The control current $i^{+}$is increased and the applied force is measured with the dynamometer table. The magnetic flux density $B$ can then calculated from equation (4).

From equation (4), $B(i)$ of a single electromagnet is assumed to be linear, in a given domain. The magnetic force model, in the linear domain, of one electromagnet is determined from equations (2) and (4) :

$$
f_{+}=\frac{4 \mu_{0} N^{2} A_{e} \cos (\alpha)}{\left(\frac{l_{e}}{\mu_{r_{s}}}+2 s_{0}\right)^{2}} i_{+}^{2}
$$




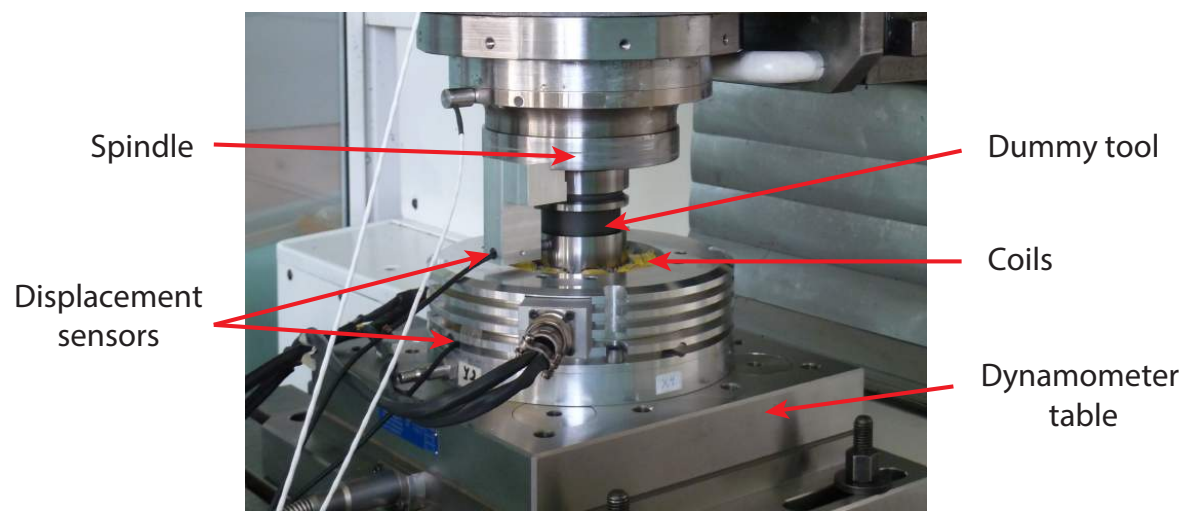

Figure 4: Experimental system

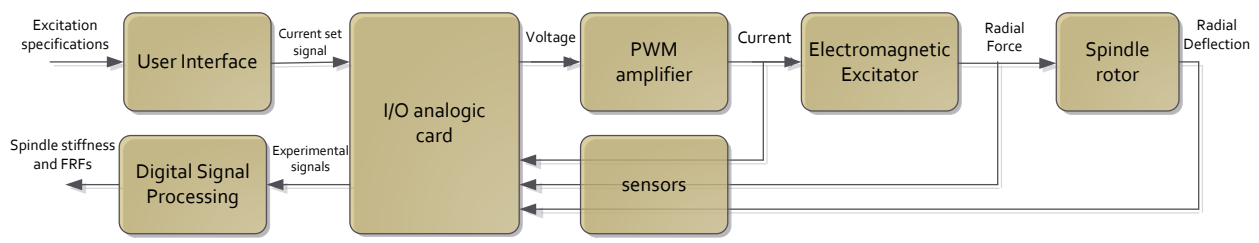

Figure 5: Experimental setup diagram

The value of the magnetic permeability is then updated from equation (10) using the parameters shown in Table 1 and the experimental results presented on figure 6a. The results presented are obtained with $\mu_{r_{s}}=7432 \mathrm{H} / \mathrm{m}$.

The figure $6 \mathrm{~b}$ present the experimental measurement of the magnetic flux density $B$ and the linear approximation obtained with the updated model. Two domain can be identified corresponding to a linear evolution of the Magnetic Flux with the current and a saturated region when $B>0.94 T$. To stay in the linear domain, the applied current must be limited to $i_{\max }=4.6 \mathrm{~A}$ in each electromagnet.

Table 1: Parameter values of the two-pole electromagnet

\begin{tabular}{lccc}
\hline \hline Term & Symbol & Value & Unit \\
\hline Effective cross-sectional area & $A_{e}$ & 182.2 & $\mathrm{~mm}^{2}$ \\
Effective magnetic path length & $l_{e}$ & 171.4 & $\mathrm{~mm}$ \\
Permeability of free space & $\mu_{0}$ & $4 \pi \times 10^{-7}$ & $\mathrm{H} / \mathrm{m}$ \\
Number of turns on a coil & $N$ & 45 & \\
Nominal air gap & $s_{0}$ & 0.25 & $\mathrm{~mm}$ \\
Pole angle & $\alpha$ & $\pi / 8$ & $\mathrm{rad}$ \\
\hline
\end{tabular}

As a consequence of this identification, the maximal reachable forces are obtained. $F_{\max }=130 \mathrm{~N}$ in linear and $190 \mathrm{~N}$ in the saturation domain. Since the signals of the two electromagnets are in phase opposition around a pre-magnetisation current $i_{0}$ (see figure 


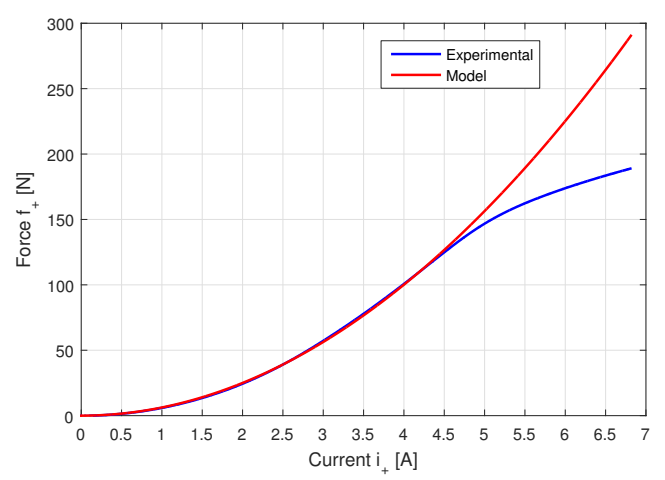

(a) Force of one electromagnet

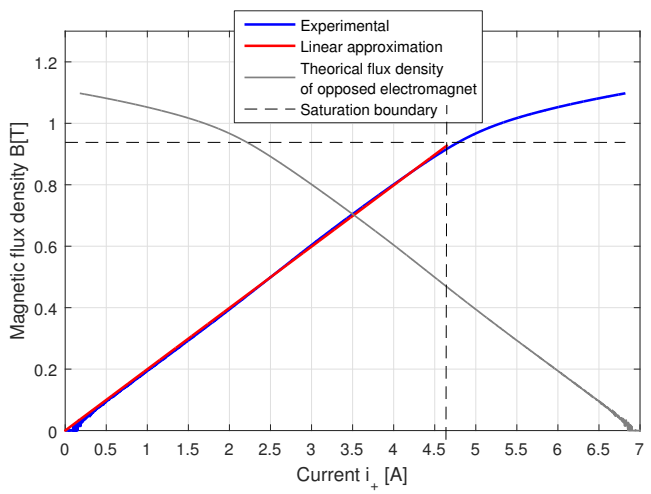

(b) Magnetic flux density

Figure 6: Experimental and simulated behavior of only one electromagnet, in relation to the coil current $i^{+}$, and identification of a limited linear domain of magnetic flux.

$6 \mathrm{~b})$ and have opposed saturation domain, the current $i_{0}$ should be chosen in the middle of the electromagnets linear domain, i.e. $i_{0}=i_{\max } / 2=3.5 \mathrm{~A}$

\subsection{Dynamic characterization and model identification}

One of the major issue to obtain FRF is to know the force level that is applied at a given frequency. Due to the low bandwidth frequency of the dynamometer table, this force level can't be measured at high frequencies $(\leq 1 \mathrm{kHz})$. To evaluate the magnetic force from equations (8) and (9), the amplitude of flux density as function of the excitation frequency $f_{e}$ must be obtained. An identical current is provided by a PWM amplifier, whatever the frequency. The link between the control current $\hat{I}$ and the magnetic flux $\hat{B}$ is therefore required. To do so, a specific experimental setup is used [15]. The operating flux density of one core can be measured through the induced voltage in the other coil. A digital oscilloscope is used to monitor and measure the RMS value of induced voltage as shown in figure 7 .

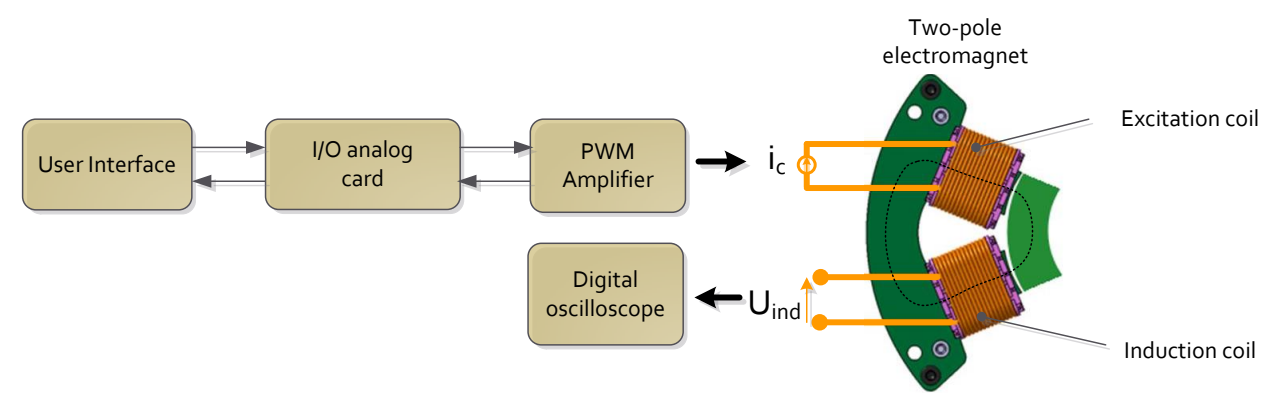

Figure 7: Experimental set-up for the determination of the magnetic flux in dynamic mode.

A sinusoidal current is supplied in the excitation coil while the induced voltage from the other coil is measured. Using Faraday's law of induction, the relation between the 
magnetic flux $\varphi$ and the RMS of induced voltage $V_{r m s}$ is given by:

$$
\varphi=\frac{V_{r m s}}{\sqrt{2} \pi f_{e} N}
$$

where $\varphi$ is the magnetic flux produced by the control current supplied to one excitation coil. Since the two-pole electromagnet consist in two identical windings, the resultant magnetic flux throughout the length of the core is produced by the total mmf of the two windings. Assuming a linear flux-mmf relationship, the total magnetic flux is that is generated by the device in dynamic mode (see 3 ) is:

$$
\phi=2 \varphi=\frac{\sqrt{2}}{\pi f_{e} N} V_{r m s}
$$

Under the assumption that the magnetic flux density is uniform across the cross section of the magnetic circuit, the magnetic flux is defined as $\phi=\hat{B} A_{e}$. It results from equation (12) that the flux density can be estimated by:

$$
\hat{B}=\frac{\sqrt{2}}{\pi f_{e} N A_{e}} V_{r m s}
$$

The amplitude of flux density in dynamic mode can hence be evaluated from equation (13) by the measure of induced voltage over the frequency range of 50 to $5000 \mathrm{~Hz}$. Figure 8 shows the amplitude of flux density versus excitation frequency for a given value of current $\hat{I}$. The optimal value of control current between the saturation boundaries of both electromagnets of an axis is $\hat{I}=1.5 \mathrm{~A}$, around the pre-magnetisation current $i_{0}=$ $3.5 \mathrm{~A}$ (see figure $6 \mathrm{~b}$ )

The eventual variation of th flux density in relation to the excitation is modeled through the dynamic relative permeability $\mu_{r_{d}}$ in equation (8). An analytical function of relative permeability as function of the frequency can be proposed to determine the amplitude of flux density from equation (8). A second order rational function is proposed, given by:

$$
\mu_{r_{d}}\left(f_{e}\right)=\frac{\sum_{i=0}^{2} a_{i} f_{e}^{i}}{\sum_{i=0}^{2} b_{i} f_{e}^{i}}
$$

The coefficients $a_{i}$ and $b_{i}$ are obtained by curve fitting experimental data shown in figure $8 \mathrm{a}$ with equation (8). Simulation of flux density and relative permeability are performed with the identified coefficient and are presented in figure 8 .

The identified model of the dynamic force, equation (9), is verified by comparing the measured and simulated frequency spectrum of the force over a frequency range of excitation from 50 to $1000 \mathrm{~Hz}$. The results illustrated in figure 9 validate the model update. It can be noticed that a dynamic force $F_{d}$ of $80 \mathrm{~N}$ can be applied until $5 \mathrm{kHz}$. The decrease of $B$ after $7 \mathrm{kHz}$ is due to the bandwidth of the PWM.

\section{Application of the excitation system}

The excitation system has been designed to analyze the quasi-static and dynamic behavior of the spindle rotor, under different spindle speeds. In this section, firstly, the 


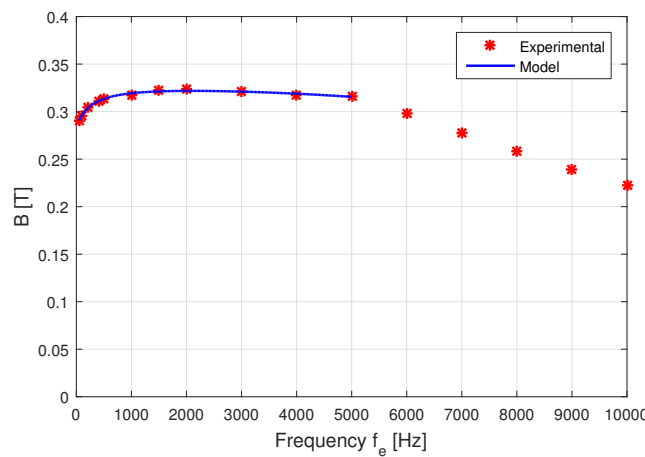

(a)

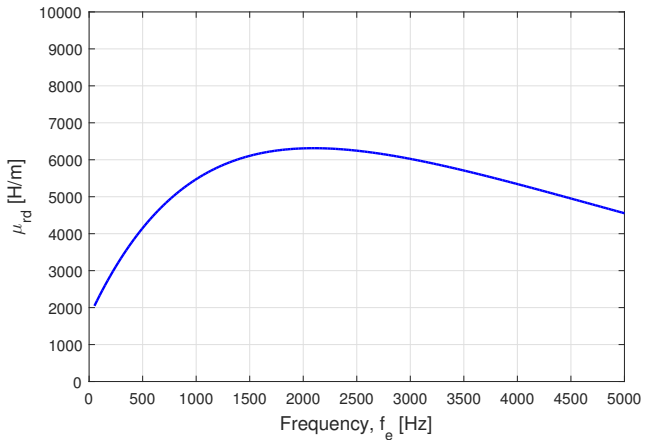

(b)

Figure 8: (a) Estimation of the flux density over frequency range of 50 to $5000 \mathrm{~Hz}$ for a control current $\hat{I}=1.5 \mathrm{~A}$. (b) Model of the dynamic relative permeability $\mu_{r_{d}}$.

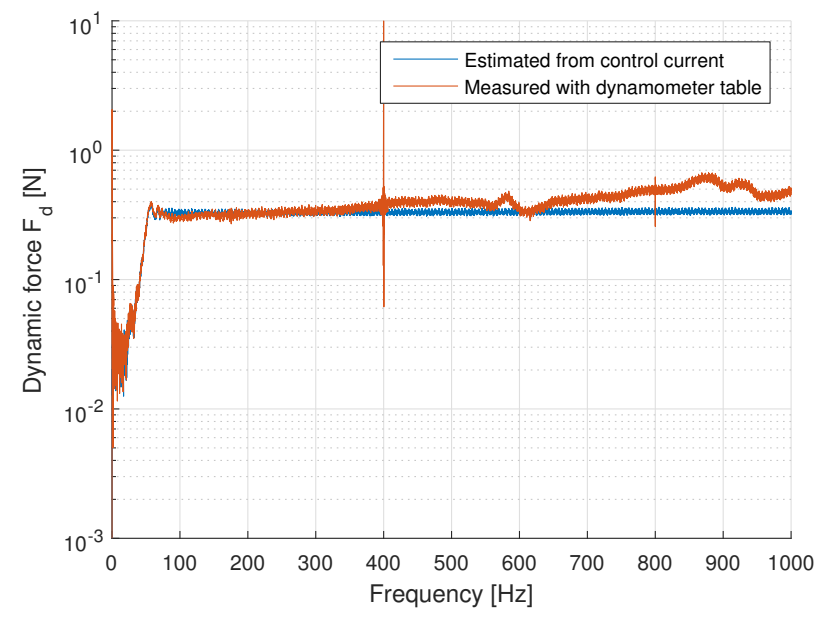

Figure 9: Comparison of measured and model-based estimated dynamic forces.

quasi-static excitation leads to the estimation of radial stiffness of the spindle (at the dummy tool tip). Particularly, the evolution of spindle stiffness with rotation speed can be observed. Then, swept sine excitation is applied for the dynamic mode and the FRF are estimated, through the dynamic force model. Lastly, the FRF evolution with spindle speed is observed.

\subsection{Quasi-static results}

The experimental set-up is the one presented in figures 3 and 4 . The rotor is initially centered in the excitation system and the electromagnets are supplied by a programmed control current in order to apply a square signal load, as shown in figure 10a. A radial force $F_{s}$ is applied to the dummy tool at different rotating speed. The resulting displacement and the applied load are measured. In order to obtain a maximum force amplitude, the pre-magnetization current is $i_{0}=3.5 \mathrm{~A}$ and maximal control current is $i_{c_{\max }}=3.5 \mathrm{~A}$. 
In this experiment, the saturated zone presented in figure 6 is reached. Therefore, the linear control of the radial force with the control current is not verified. The static force model was developed for the linear domain and cannot be used here, out of the linear domain. Consequently, the force is directly measured with the dynamometer table and not estimated from the current measurement.

The force and displacement measurements data are filtered to remove the signals noise. The selected cutoff frequency is low and thus eliminates the tool runout noise and high frequency noise from the displacement signal. The filtered signal is shown for four different speeds in figure 10b. The assumption of a negligible displacement of the dummy-tool compared to the initial air-gap $s_{0}=250 \mu \mathrm{m}$ is verified from the quasi-static measurement.

As a result, the evolution of the radial stiffness of the spindle is obtained from the quasi-static test presented (see figure 11). It is mainly due to the centrifugal forces on bearing balls that modifies the contact angle and radial stiffness.

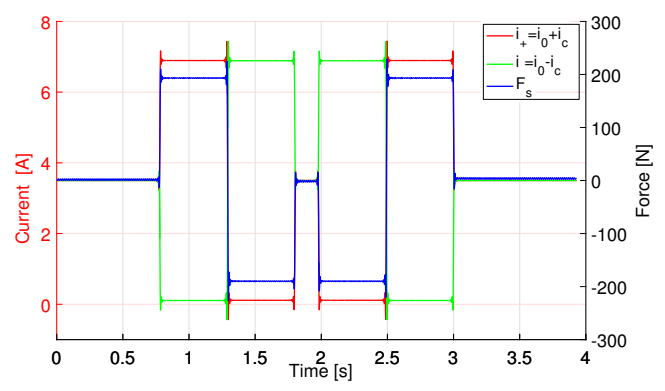

(a) Excitation currents and radial force.

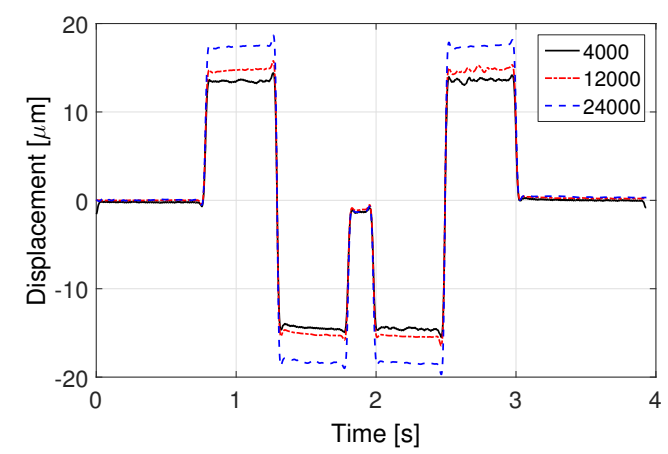

(b) Displacements at different spindle speeds $N$ [rpm].

Figure 10: Measurements in quasi-static mode.

\subsection{Dynamic validation}

The dynamic validation of the excitation system consists in accessing the FRF at the dummy tool tip by exciting the rotor over a frequency range of 50 to $5000 \mathrm{~Hz}$. The experimental setup corresponds to figures 3 and 4 . The FRFs are estimated for different spindle speeds. The magnetic force applied to the rotor in the x-direction is evaluated from equation (9) and from the measure of the control current $i_{c}$.

\subsubsection{Swept sine control current}

In order to obtain a FRF over a specified frequency range, the excitation current is carried out by a sine sweep with a linearly varying frequency. The control current is described by:

$$
i_{c}=\hat{I} \sin \left[2 \pi t\left(\frac{f_{\max }-f_{\min }}{2 T} t+f_{\min }\right)\right]
$$




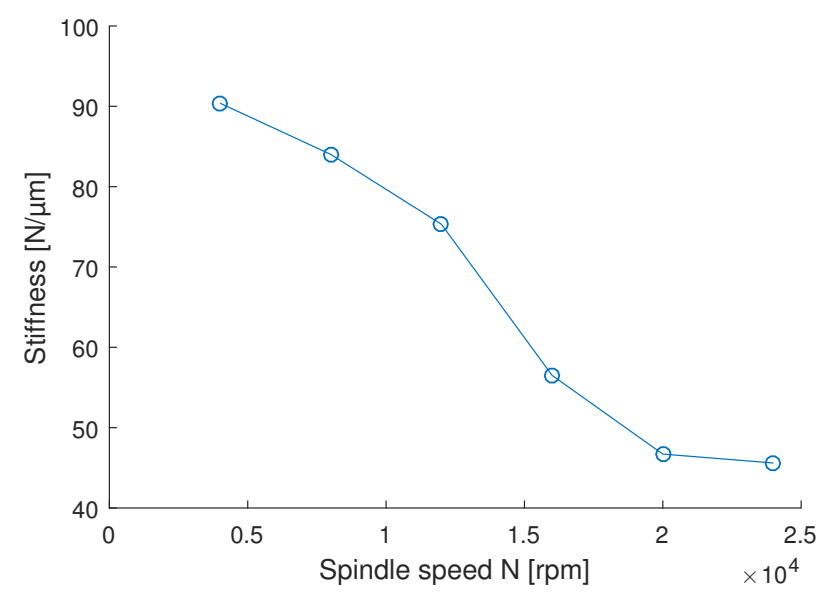

Figure 11: Variation of the spindle radial stiffness (at tool tip) with rotation speed.

where $f_{\min }$ and $f_{\max }$ are respectively the minimum and maximum frequencies and $T$ is the duration of the dynamic test in seconds. The time series of a swept sine between 0 and $10 \mathrm{~Hz}$ are shown in figure 12a. The frequency spectrum of the signal is given in figure $12 \mathrm{~b}$.

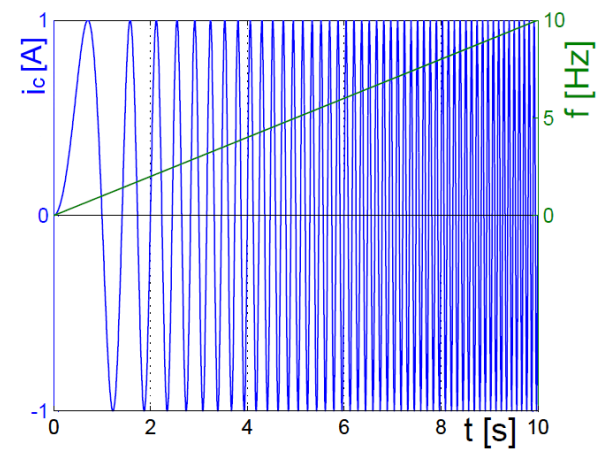

(a) Time series

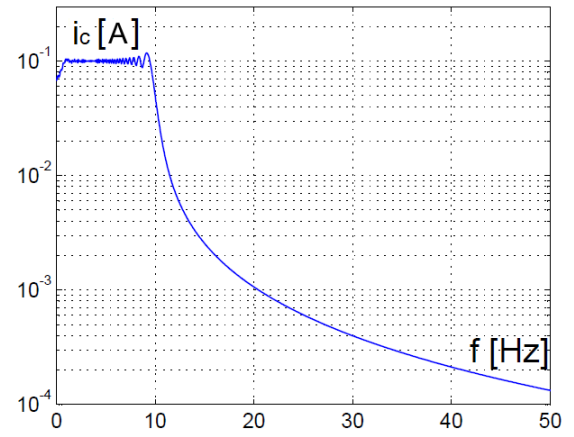

(b) Frequency spectrum

Figure 12: Example of a swept sine excitation from 1 to $10 \mathrm{~Hz}$ for $\hat{I}=1 A$.

As shown in figure $12 \mathrm{~b}$, a swept sine solicitation excites all frequencies between $f_{\min }$ and $f_{\max }$. The experimental dynamic test of spindles are performed with a sweep duration of 30 seconds in the frequency range $50-4000 \mathrm{~Hz}$.

\subsubsection{Signal processing}

A specific signal processing is necessary for measured displacements. After a low-pass filter to suppress the high frequency noise, it is necessary to suppress the displacements corresponding to the tool runout. This is observed in the frequency domain by contributions at harmonics of the shaft frequency (see raw signal in figure 13). The filtered 
signal is obtained after the use of an $\mathrm{H} 1$ formulation to compute the FRF (i.e. cross spectrum of the displacement and load/autospectrum of the load). This formulation is used to reduce the noise on the displacement signal. The Matlab smooth function with the "rloess" option is then used to suppress the undesired harmonic content of the frequency domain.

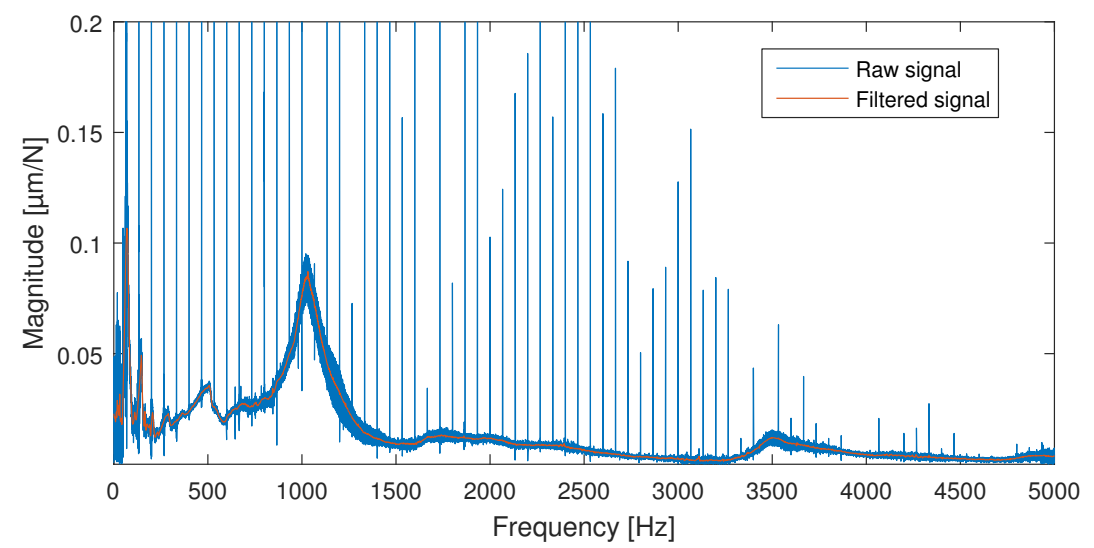

Figure 13: Estimated FRFs in frequency domain with and without post-processing.

\subsubsection{Experimental dynamic results}

Dynamic measurements were performed at different spindle speeds. Figure 14 shows the experimental results after post-processing. The modes frequencies and amplitudes varies with spindle speeds, which justify the necessity to use such a device. It is due to the evolution of the dynamic behavior of the spindle at high speed. A validation tap test was performed to confirm the FRF prediction made with the electromagnetic excitation device. It is plotted in dashed line in the figure and it is in good agreement with the FRF measurement made with the electromagnetic excitation device at $0 \mathrm{rpm}$. Discrepancies can be seen at high frequencies (higher than $2 \mathrm{kHz}$ ) and are due to limitations of tap test configuration.

\section{Conclusion}

A specific device has been developed to analyze the quasi-static and dynamic behavior of HSM spindles. Force models are developed both for static and dynamic modes. Besides, a method to characterize the electromagnetic system behavior in static and dynamic mode is presented in this paper. The limit between the linear and saturation domain of the electromagnets has been experimentally identified; as well as the static and dynamic relative permeabilities that are required for the force models. Maximum forces of $\pm 190 \mathrm{~N}$ for the static mode and $\pm 80 \mathrm{~N}$ for the dynamic mode up to a frequency of $5 \mathrm{kHz}$ were obtained. Quasi-static and dynamic measurement were performed at up to 24000rpm on an industrial spindle to illustrate the system performance. The approach was also validated by a tap test measurement of FRF. Consequently, the electromagnetic system, remains a highly efficient tool to analyze the dynamic behavior of a machine-tool spindle. 

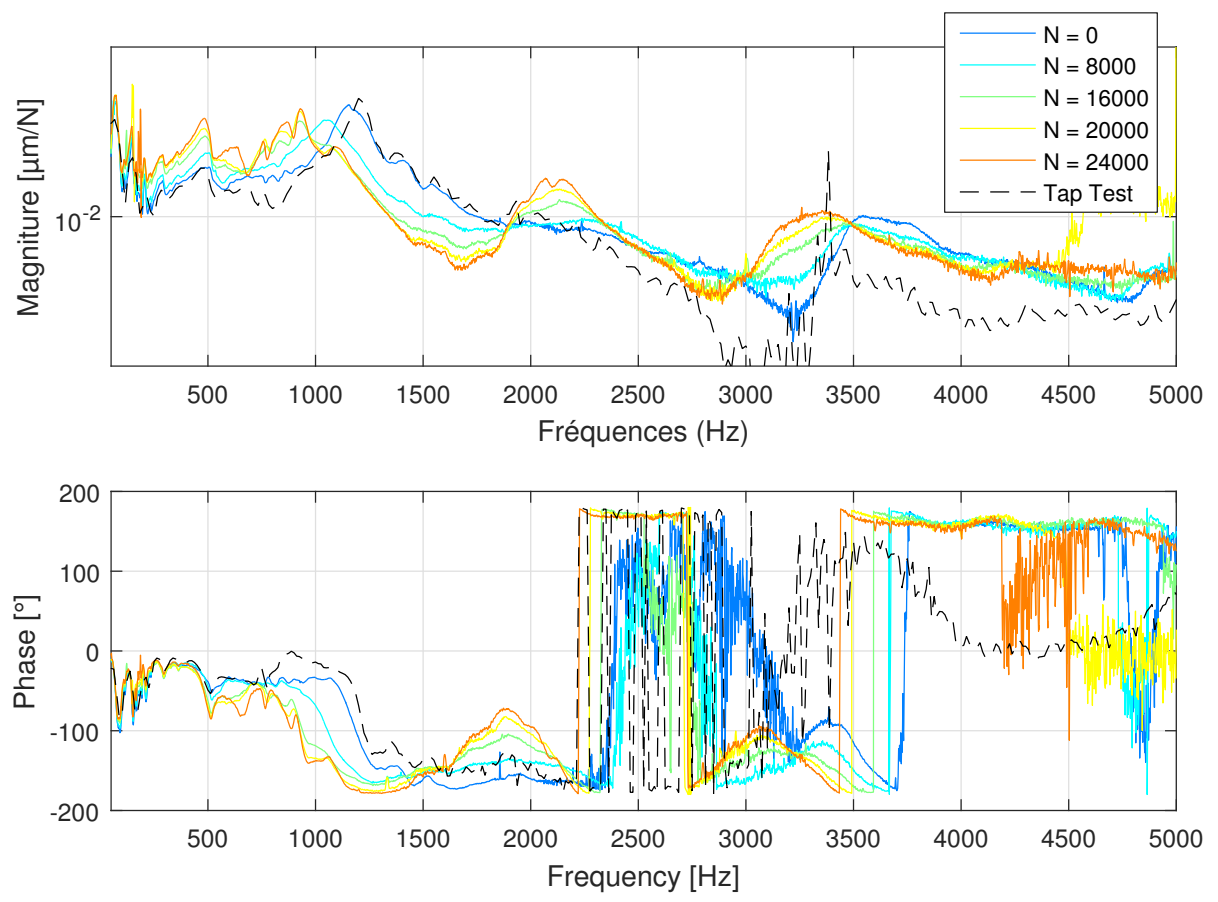

Figure 14: FRF at the dummy tool tip in relation to spindle speed $N$ [rpm].

\section{Acknowledgments}

The research was conducted within the context of the French FUI project "QuaUsi". The authors would like to thank the company Europe Technologies and Precise where some experiments were made.

\section{References}

[1] C. De Castelbajac, M. Ritou, S. Laporte, B. Furet, Monitoring of distributed defects on HSM spindle bearings, Applied Acoustics 77 (2014) 159-168. doi:10.1016/j.apacoust.2013.07.008. URL http://dx.doi.org/10.1016/j.apacoust.2013.07.008

[2] M. Ritou, S. Garnier, B. Furet, J. Hascoet, Angular approach combined to mechanical model for tool breakage detection by eddy current sensors, Mechanical Systems and Signal Processing 44 (1-2) (2013) 211-220. doi:10.1016/j.ymssp.2013.02.004. URL http://linkinghub.elsevier.com/retrieve/pii/S0888327013000836

[3] Y. Cao, Y. Altintas, A General Method for the Modeling of Spindle-Bearing Systems, Journal of Mechanical Design 126 (6) (2004) 1089. doi:10.1115/1.1802311.

[4] A. Albrecht, S. S. Park, Y. Altintas, G. Pritschow, High frequency bandwidth cutting force measurement in milling using capacitance displacement sensors, International Journal of Machine Tools and Manufacture 45 (9) (2005) 993-1008. doi:10.1016/j.ijmachtools.2004.11.028.

[5] C. Rabréau, D. Noel, S. Le Loch, M. Ritou, B. Furet, Phenomenological model of preloaded spindle behavior at high speed, International Journal of Advanced Manufacturing Technologydoi:10.1007/ s00170-016-9702-1.

[6] T. L. Schmitz, J. C. Ziegert, C. Stanislaus, A method for predicting chatter stability for systems with speed-dependent spindle dynamics, Papers Presented at NAMRC 32 Jun 14200432 (2004) 17-24. 
[7] C. Knospe, Active magnetic bearings for machining applications, Control Engineering Practice.

[8] E. Abele, M. Kreis, M. Roth, Electromagnetic Actuator for in Process Non-Contact Identification of Spindle-Tool Frequency Response Functions, in: CIRP 2nd International Conference on High Performance Cutting, Vancouver, 2006.

[9] S. Auchet, P. Chevrier, M. Lacour, P. Lipinski, A new method of cutting force measurement based on command voltages of active electro-magnetic bearings, International Journal of Machine Tools and Manufacture 44 (14) (2004) 1441-1449. doi:10.1016/j.ijmachtools.2004.05.009.

[10] M. Rantatalo, J.-O. Aidanpää, B. Göransson, P. Norman, Milling machine spindle analysis using FEM and non-contact spindle excitation and response measurement, International Journal of Machine Tools and Manufacture 47 (7-8) (2007) 1034-1045. doi:10.1016/j.ijmachtools.2006.10.004

[11] A. Matsubara, T. Yamazaki, S. Ikenaga, Non-contact measurement of spindle stiffness by using magnetic loading device, International Journal of Machine Tools and Manufacture 71 (2013) 20-25. doi:10.1016/j.ijmachtools.2013.04.003.

[12] S. Lopez, B. Cassoret, J. Brudny, L. Lefebvre, J. Vincent, Grain oriented steel assembly characterization for the development of high efficiency ac rotating electrical machines, IEEE Transaction on Magnetics 45 (10).

[13] G. Schweitzer, Active magnetic bearings - chances and limitations, In Proceedings of the 6th International IFToMM Conference on Rotor Dynamics.

[14] A. Balakrishnan, W. T. Joines, T. G. Wilson, Air-gap reluctance and inductance calculations for magnetic circuits using a schwarz-christoffel transformation, IEEE Transactions on Power Electronics 12 (4) (1997) 654-663. doi:10.1109/63.602560.

[15] H. W. Lamsont, A Method of Measuring the Magnetic Properties of Small Samples of Transformer Laminations * (1940) 541-548. 